\title{
High-capacity spatial contextual memory
}

\author{
YUHONG JIANG, JOO-HYUN SONG, and AMANDA RIGAS \\ Harvard University, Cambridge, Massachusetts
}

\begin{abstract}
Humans show implicit memory for complex spatial layouts, which aids in subsequent processing of these layouts. Research efforts in the past 5 years have focused primarily on a single session of training involving a dozen repeated displays. Yet every day, people encounter many more visual layouts than were presented in such experiments. In this study, we trained subjects to learn 60 repeated displays, randomly intermixed within 1,800 nonrepeated displays, spread over 5 consecutive days. On each day, the subjects conducted visual search on 360 new displays and a new set of 12 repeated displays, each repeated 30 times. Contextual memory was observed daily. One week after the fifth session, the subjects still searched faster on the repeated displays learned previously. We conclude that the visual system has a high capacity for learning and retaining repeated spatial context, an ability that may compensate for our severe limitations in visual attention and working memory.
\end{abstract}

Humans process a visual display more efficiently when they encounter it for a second time. A previously perceived object, now presented very briefly, is correctly identified more accurately than a new object, showing priming (Tulving \& Schacter, 1990). Such perceptual facilitation is seen not only for isolated shapes or words, but also for complex visual displays (Chun \& Jiang, 1999; Fiser \& Aslin, 2001). When asked to count an array of random dots, observers are faster at counting a display presented before (Lassaline \& Logan, 1993), even when they do not have explicit memory for the display. When conducting visual search for a $\mathrm{T}$ target presented among $\mathrm{L}$ distractors, observers are faster at detecting the target when the same display is occasionally repeated, even when the repeated displays are not easily discriminated from the new ones (Chun \& Jiang, 1998). Our ability to retain complex visual information in implicit memory is remarkable, considering our limitations in explicit visual memory. Recent studies using the change detection paradigm (Rensink, 2002) suggest that humans are quite poor at detecting large-scale changes in natural viewing and social interactions (Levin \& Simons, 1997; Rensink, O’Regan, \& Clark, 1997; Simons \& Levin, 1998). Yet, in everyday life, we negotiate our visual environment with ease, perhaps because implicit visual learning allows us to retain visual information for future use. This study tests the durability and capacity of spatial contextual memory.

To test humans' ability to learn repeated spatial context, Chun and Jiang (1998) examined how repeated presentation of a search display facilitated performance. Subjects were asked to search for a $\mathrm{T}$ target presented

This research was supported by ARO LS-46926 and NIH MH071788. We thank Sidney Burks for data collection and Nelson Cowan, James Cutting, Arthur Glenberg, James Nairne, Gill Rhodes, and Daniel Simons for suggestions. Correspondence should be addressed to Y. Jiang, Department of Psychology, Harvard University, 33 Kirkland Street, WJH 820, Cambridge, MA 02138 (e-mail: yuhong@wjh.harvard.edu). among $\mathrm{L}$ distractors. The spatial layout of the search elements defined the global visual context for the target. Subjects were tested in two conditions: old and new. In the old condition, displays were repeated once every two dozen trials or so. Because both the target's location and distractor layout repeated, the old condition contained consistent associations between the distractors and target locations. In contrast, in the new condition, only the target locations occasionally repeated, whereas distractor locations did not. The experiment was divided into 30 blocks, each of which contained several unique old displays randomly intermixed with the presentation of new displays. Results showed that as the experiment progressed, search speed became faster on old displays than on new ones. We call this context-specific learning effect contextual cuing. It is as if the context of the target guided, or cued, visual attention to the position of the target (Chun \& Jiang, 1998).

To examine whether contextual cuing is explicit or implicit, at the end of a 1-h session, Chun and Jiang (1998) showed subjects old and new displays. This time, subjects were asked to recognize the old displays. Despite the large learning effect revealed by search speed, subjects were completely at chance in explicit recognition. They lacked any explicit knowledge of which displays were old and which were new, and furthermore could not guess the target location for any given old display (Chun \& Jiang, 2003). Contextual cuing is fast, with an effect appearing after only five or six repetitions; it is strong, preserved even when only half of the items are repeated; and it is implicit, freeing limited awareness to other mental processes (Chun \& Jiang, 1998).

Contextual visual learning is also shown when a target shape is consistently paired with a group of distractor shapes (Chun \& Jiang, 1999; Endo \& Takeda, 2004; Fiser $\&$ Aslin, 2001). In addition, subjects searching for multiple moving elements become faster if the target's motion trajectory is consistently associated with repeated 
distractor trajectories (Chun \& Jiang, 1999; Olson \& Chun, 2001). The power and ubiquity of contextual cuing may compensate for our severe limitations in visual attention and working memory.

Surprisingly, research efforts in the past 5 years have focused primarily on a single session of training involving a dozen repeated displays. Yet, in everyday activities, people learn many more visual layouts. When we walk into a new environment, there is a constant need to acquire new information. How many visual displays can be acquired through implicit visual learning? How will previously acquired memory interfere with future learning and vice versa? Learning and memory of different displays acquired in successive training sessions may interact by producing proactive or retroactive interference (Anderson \& Neely, 1996; Crowder, 1976). Testing subjects on multiple training sessions can reveal these interference effects.

\section{PRESENT STUDY}

Twelve subjects were tested in five training sessions, spread over 5 consecutive days. In each session, the subjects performed a visual search task in 30 blocks, searching for a $\mathrm{T}$ among Ls. Each block contained 24 different displays: 12 were old displays, presented once per block for a total of 30 times, and 12 others were new displays, not repeated. Across the five sessions, different old and new displays were tested, resulting in a total of 60 old displays (12 per day) and 1,800 new displays (360 per day). One week after the last training session, the subjects returned to the lab and were tested on all 60 old displays, randomly intermixed with 60 new displays that were not presented previously. The final testing session was included to assess whether there was long-term retention of displays learned in multiple sessions. This design addresses several questions about spatial contextual learning.

\section{Capacity}

Previous studies on contextual cuing show that humans are capable of learning 12 repeated displays, each presented 24-30 times, among 300 new displays (e.g., Chun \& Jiang, 1998). Given that we encounter many more than 12 displays every day, it is of theoretical and empirical significance to determine whether one can learn a much larger number of repeated displays. In this study, we selected an arbitrarily large number-60 repeated displays presented among 1,800 new displays - to determine whether this number is within the capacity of spatial contextual learning.

\section{Proactive and Retroactive Interference}

Proactive interference refers to how present learning interferes with the ability to learn in the future, whereas retroactive interference refers to how present learning interferes with the retrieval of memory acquired in the past (Crowder, 1976). In our study, displays learned on each day could produce proactive interference on later days. So learning on Day 5 would suffer from the most proactive interference. Conversely, displays learned on later days could produce retroactive interference for memory of displays learned earlier, such that when assessed 1 week later, memory for the displays of Day 1 would suffer from the most retroactive interference. Thus, if there is a significant proactive interference effect, learning on Day 5 should be the weakest, and if there is a significant retroactive interference effect, retention of the leaning on Day 1, assessed 1 week after all sessions are complete, should be the poorest. A similar logic has been used previously to study interference effects in word memory (Jacoby, 1983).

Proactive and retroactive interference are critical features of human learning and memory (Anderson \& Neely, 1996; Crowder, 1976). The degree of interference depends on the similarity between the learned materials. The more similar different memory lists are, the larger the degree of interference (Lustig \& Hasher, 2001a; Osgood, 1953). Given that the 60 repeated and 1,800 nonrepeated displays tested in this study were generated at random by a similar algorithm, they constituted a highly similar series of displays and were more homogeneous than natural scenes we encounter everyday. Using these stimuli thus provided us with a good opportunity to observe proactive and retroactive interference.

\section{Long-term Retention}

A high-capacity memory system is of limited use if it is short lived. Amnesiac patients, lacking the ability to transcribe immediate memory to explicit long-term memory, are confined to the present moment, unable to use declarative memory acquired in the past (Schacter, 1996). Similarly, the function of spatial contextual memory would be limited if it were short lived. Yet after having encountered 60 repeated displays and 1,800 nonrepeated ones, subjects now face a difficult task of matching a current display with their memory. If subjects continue to benefit from previous learning 1 week after the training sessions, it would suggest the presence of an extraordinary learning mechanism.

In sum, by training subjects on 5 consecutive days and testing them 1 week later, this study allows us to assess the capacity of spatial contextual learning, its long-term durability, and proactive and retroactive interference effects among patterns learned on separate days.

\section{METHOD}

\section{Subjects \\ Twelve subjects ( 9 females and 3 males; mean age 23 years) from Harvard University volunteered for this experiment. All had nor-} mal or corrected-to-normal visual acuity.

\section{Equipment}

The subjects were tested individually in a room with normal interior lighting. They viewed a computer screen from an unrestricted distance of about $57 \mathrm{~cm}$, at which distance $1 \mathrm{~cm}$ corresponded to $1^{\circ}$ visual angle. 


\section{Sessions}

Each subject was tested individually in six sessions. Sessions $1-5$ were training sessions occurring on 5 consecutive days (one session per day); Session 6 was a final testing session. There was a 1week interval between Sessions 5 and 6 (range: 6-10 days, $M=$ 7.3). The subjects were tested at approximately the same time on each day.

Sessions 1-5. The first five sessions were similar in design. On each day, the subjects received 100-120 trials of practice on nonrepeated visual search displays. Then they participated in the experimental session, performing a visual search task for 30 blocks of 24 trials each. They searched for a white $\mathrm{T}\left(1^{\circ} \times 1^{\circ}\right)$ presented among 15 white Ls $\left(1^{\circ} \times 1^{\circ}\right)$. The target was presented on every trial, but was rotated either to the left or to the right. The subjects were asked to press the left or right key, corresponding to the target's direction of rotation. The items were presented on a gray background, positioned randomly on an invisible $10 \times 10$ matrix $\left(24^{\circ} \times 24^{\circ}\right)$. Figure 1 shows a sample display.

Each block contained 24 different search displays: 12 old and 12 $n e w$, randomly intermixed together. Since the old displays were presented once per block, the subjects searched from such displays 30 times by the end of the session. The new displays were newly generated in each block, with the constraint that the target's location was repeated across blocks. Thus, although the target's location in the new condition had repeated 30 times in the session, it was presented within a new spatial layout every time a subject saw it.

It is important to note that entirely different displays were presented on different days. Thus, 12 old displays were repeated 30 times on Day 1, and 12 other displays were repeated 30 times on Day 2, and so on. The old displays were thus "new" at the beginning of each session and became "old" in each session with repetitions. The training sessions across days were thus comparable, except that the learning on Day 1 might interfere with that on Days 2-5, the learning on Day 2 might interfere with that on Days 3-5, and so on.

Session 6. One week after Session 5, the subjects returned to the lab and participated in a testing session. The experimental phase contained all 60 old displays and 60 new ones. The new displays were generated anew in Session 6 and were yoked controls of the old displays. That is, to control for learning of the old target locations per se, the new displays shared the same target locations as the old ones'. The two types of displays were randomly intermixed to produce a block of 120 trials. We repeated all 120 trials once, producing 24 trials per day per condition.

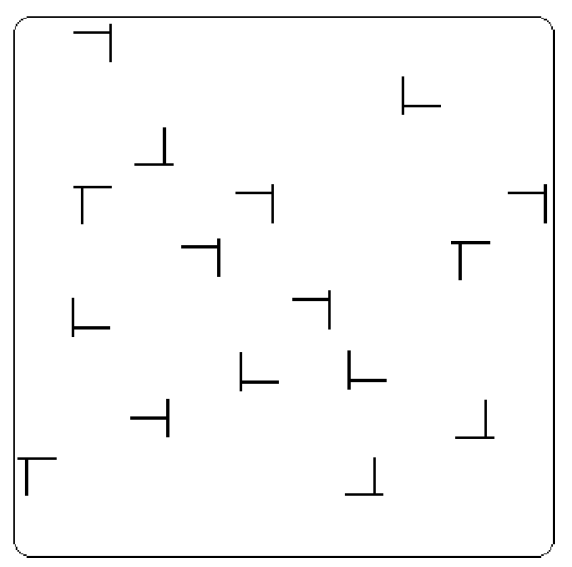

Figure 1. A schematic of a trial tested in this study. The subjects searched for a rotated $T$ target presented among $L$ distractors. They pressed the left or right key to report the target's direction of rotation.
Trial. Each trial started with a fixation point $\left(0.3^{\circ}\right)$ that lasted $400 \mathrm{msec}$, followed by the search display. Once the subjects made a response (left or right keypress), the search display was erased. An incorrect response was followed immediately by a "sad face" icon. The next trial started $1 \mathrm{sec}$ later. The subjects were allowed to take a break after each block and proceeded at their own pace.

\section{RESULTS}

We calculated mean reaction time (RT) for each subject. ${ }^{1}$ RT gradually improved across the 5 days, even for nonrepeated displays. This reflects general skill learning in the visual search task (Schneider \& Shiffrin, 1977), rather than specific learning of the repeated spatial context. The reduction in RT in the baseline condition (nonrepeated trials) makes the comparison of contextual cuing across days more convoluted (Chapman, Chapman, Curran, \& Miller, 1994). For example, because the RT on Day 1 was much longer than that of Day 5 , there could be a numerically larger contextual learning on Day 1 than on Day 5. Therefore, we used two indexes of spatial memory: RT difference and percentage saving. RT difference is measured as the difference in mean RT between the nonrepeated and repeated displays in blocks 11-30. ${ }^{2}$ Percentage saving is the ratio between the RT difference and the mean RT in the nonrepeated condition in blocks 11-30, which were used because contextspecific learning had emerged. These two measures produce similar patterns of data. In the following results, we shall report both.

\section{Sessions 1-5}

Accuracy in all sessions was high ( $97 \%$ or higher) and was not significantly influenced by condition (new vs. old ) or block (1-30); all $p s>.15)$. We calculated the mean RT for each subject from correct trials only. Figure 2 shows the group mean in all five training sessions. Note that a different set of 12 old displays was shown every day, so there should be no difference between the new and old conditions at the beginning of each session.

An analysis of variance (ANOVA) on condition (repeated vs. nonrepeated) and block (1-30) was conducted for each session's mean RT separately. ${ }^{3}$ This analysis revealed a significant main effect of block in all sessions $[F(29,319)=4.71, p<.0001$ for Session $1 ; F=3.15$, $p<.0001$ for Session $2 ; F=2.37, p<.0001$ for Session $3, F=1.90, p<.004$ for Session 4 , and $F=1.53$, $p<.04$ for Session 5], suggesting that subjects' RT became significantly faster as the experiment progressed on all days. The main effect of condition was significant on Day $1[F(1,11)=11.26, p<.006]$, Day $3(F=12.64$, $p<.005)$, Day $4(F=8.02, p<.016)$, and Day $5(F=$ $11.20, p<.007)$, but was not significant on Day $2(F=$ $1.76, p<.20)$. The interaction between condition and block was significant on Day $1[F(29,319)=1.88, p<$ $.006]$, Day $3(F=1.60, p<.023)$, and Day $5(F=1.69$, $p<.017)$, but not significant on Days 2 and $4\left(F_{\mathrm{s}}<\right.$ $1.45, p s>.25) .^{4}$ 


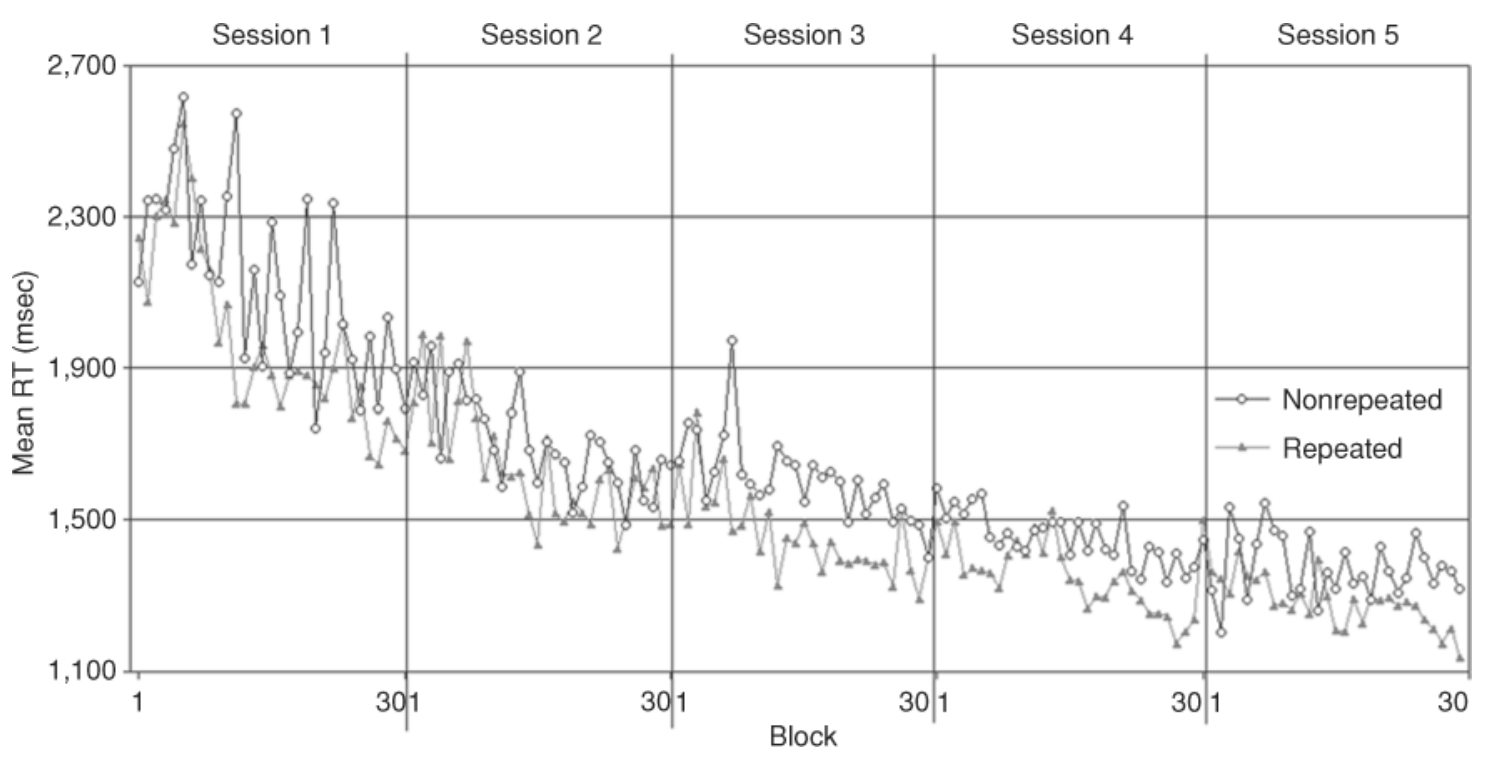

Figure 2. Mean RT from the five training sessions. Each session included 12 repeated displays, repeated once per block for 30 blocks, for a total of 360 nonrepeated displays. The target locations were repeated in both repeated and nonrepeated displays. Data were collected from the same group of 12 subjects tested on 5 consecutive days.

The size of contextual cuing, calculated as the difference in mean RT between the new and old conditions in Blocks 11-30, was $205 \mathrm{msec}, 83 \mathrm{msec}, 161 \mathrm{msec}, 107 \mathrm{msec}$, and $100 \mathrm{msec}$, for Days $1-5$, respectively. They were significantly greater than zero $[F(1,11)=52.88, p<.001]$, but not significantly different from one another $[F(4,44)=$ $1.27, p>.25]$. When these values were scaled against the RT in the new condition, the percentage saving of contextual cuing was $10 \%, 4 \%, 11 \%, 7.4 \%$, and $7.2 \%$, for Days $1-5$, respectively. These values were significantly greater than zero $[F(1,11)=48.19, p<.01]$, but not significantly different from one another $[F(4,44)=$ $1.26, p>.30]$.

Thus, over a 5-day period, subjects were able to learn 60 repeated displays from a total of 1,800 nonrepeated displays. Learning was observed in all days, although it was statistically weak in Day 2. Although we cannot rule out proactive interference, such interference must be relatively weak. For example, when subjects started the experiment on Day 5, they had already been exposed to 48 old displays, 1,440 new displays, and 500 practice displays on the previous 4 days. Yet they were still able to quickly learn a different set of 12 repeated displays from 360 nonrepeated displays. This suggests that contextual cuing is relatively insensitive to proactive interference.

Individual differences and reliability. Different subjects had different search speeds and showed different degrees of contextual cuing. To assess the reliability of such individual differences, we tested the correlation of performance across the 5 days. If a given subject was consistently fast in all sessions, correlation for RTs in the baseline condition (the nonrepeated displays) would be high. This was indeed what we found. There were 10 pairwise Pearson's correlations for mean baseline RTs across the 5 days. They ranged from .56 to .95 . When these values were tested against 0 in a one-sample $t$ test, they were significantly greater than zero $[t(9)=19.64, p<.0001]$. A similar correlation analysis on the size of contextual cuing (new-old in Blocks 11-30), however, showed pairwise Pearson's $r$ s ranging from -.35 to .29 . The 10 pairwise correlations were not significantly different from zero $[t(9)=-.72, p>.45]$. Similarly, the percentage saving of contextual cuing showed no correlation within an individual, with Pearson's $r$ s ranging from -.32 to .22 $[t(9)=-.34, p>.70] .5$ Thus, individuals who showed a large contextual cuing effect on one day might not show a large effect on another day, and vice versa. Individual differences in contextual cuing measured in a single session appeared to reflect primarily random noise. There was no evidence that some individuals always showed a large learning effect and others always showed a small learning effect.

\section{Session 6}

One week after the last training session, subjects returned to the lab and conducted visual search among 60 old displays and 60 new ones. The old displays were the same as those learned in Sessions 1-5. The new displays were yoked controls that contained repeated target locations learned in previous sessions, but newly generated distractor locations. Figure 3 shows the mean RT data, separately for the new and old conditions and training days. ${ }^{6}$

An ANOVA on condition (new vs. old in previous sessions) and session (1-5) revealed a significant main effect of condition $[F(1,11)=17.57, p<.002]$. The main effect of session was not significant $(F<1)$, nor was the interaction between condition and session $(F<1)$. An ad- 


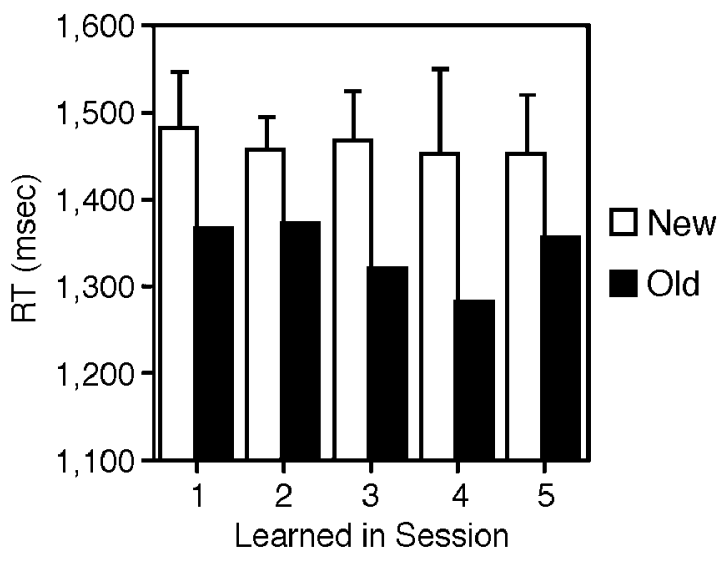

Figure 3. Results from Session 6. Displays learned from Sessions 1-5 were presented again in Session 6; otherwise, the new and old displays were comparable in Session 6. Subjects were faster at visual search from displays learned before, showing long-term retention. Error bars show standard errors of the difference between new and old conditions.

ditional ANOVA restricted to the old condition showed no effect of session $[F(4,44)<1]$. Note that the only difference between new and old conditions was that the old displays were previously repeated a week earlier. Still, subjects were faster at searching from the old displays, which suggests that they had retained previous learning for at least a week. The saving effect was seen for displays learned from all five sessions: $114 \mathrm{msec}$ for displays learned in Session $1[t(11)=3.22, p<.008], 82 \mathrm{msec}$ for displays learned on Session $2[t(11)=2.57, p<$ .026], $144 \mathrm{msec}$ for displays learned on Session 3 $[t(11)=2.40, p<.035], 171 \mathrm{msec}$ for displays learned on Session $4[t(11)=1.85, p<.09]$, and $94 \mathrm{msec}$ for displays learned on Session $5[t(11)=1.59, p<.14$, all $p$ s two-tailed]. Note that because the prediction about memory saving was unidirectional, a one-tailed test would be justified, in which case, saving from all days was marginal or significant. Interestingly, although contextual cuing was not statistically significant during the training on Day 2, the long-term retention of the patterns on Day 2 was significantly better than chance. This suggests that the subjects had originally learned the displays on Day 2.

\section{DISCUSSION}

By training subjects on different displays for 5 consecutive days and testing for long-term retention of all learned displays 1 week later, we have shown here a very high capacity for spatial context learning. Let us consider what it means to retain contextual cuing in Session 6. In order to search from an old display faster than a new one, subjects needed to (1) successfully match the current display with the memory trace of that display (and not the other ones), established a week earlier, and (2) to access the associated target location once a match is found
(Logan, 1988). Both steps had to be completed within about $1 \mathrm{sec}$ in order to enhance RT. Given that subjects had previously seen nearly 2,000 displays similar to the display currently shown on the screen, it is extraordinary that they could still make the match. Such a high-capacity spatial learning mechanism is certainly not just for learning arbitrary $\mathrm{T}$ and $\mathrm{L}$ displays as tested here. It reflects the power of the visual statistical learning mechanisms.

A second finding from this study is that contextual cuing was relatively insensitive to proactive and retroactive interference. A significant contextual cuing effect was observed on later training sessions, after subjects had already been exposed to many similar visual displays. This suggests that subjects were still capable of acquiring new learning, whether or not they had previously been trained on other displays. In addition, displays learned on Day 1 were still retained on Session 6 tested 12 days later, during which many displays were learned. This suggests that retroactive interference, if there was any, did not completely disrupt previous memory. This is not to deny that there might be some, undetected, proactive or retroactive interference. Previous research suggests that implicit memory is not immune to proactive and retroactive interference effects when two memory items compete for response to a memory cue (Lustig \& Hasher, 2001b). The principle of response similarity also applies to visual contextual cuing. When a given repeated display is associated with two potential target locations, contextual cuing is cut in half (Chun \& Jiang, 1998). However, the present study shows that as long as a given repeated display is always associated with one target location, contextual cuing is relatively resistant to interference effects.

What is the nature of such contextual memory: Is it implicit or explicit? Previous studies using the same procedure-repeating 12 old displays 30 times in a session - found that subjects were unable to recognize the old displays (Chun \& Jiang, 1998, 2003). Such a lack of awareness is understandable, given that the repeated displays were highly similar to nonrepeated ones. Although we did not directly test explicit recognition of the displays, it is likely that subjects in the present study also lacked explicit awareness of the repetition. Nonetheless, we wish to emphasize the presence of a cuing effect, rather than its implicit nature. From a functional point of view, contextual cuing does not have to be implicit since performance is enhanced whether or not learning reaches awareness. What our study has shown is a surprisingly robust form of spatial contextual learning that persists under conditions of potential proactive and retroactive interference.

In summary, we trained subjects on five consecutive sessions of contextual cuing and found that they were able to acquire spatial contextual learning for as many as 60 repeated displays, presented within 1,800 nonrepeated displays. Learning acquired from one training session showed long-term retention, such that the old displays were still searched faster than new ones when 
tested 1 week later. We have shown here humans' surprisingly high capacity in learning complex visual contexts. Our study underscores the visual system's efficiency at statistical learning and resonates with Kolers's claim that humans have excellent pattern-analyzing memory (Kolers, 1976). Kolers reached this conclusion by showing that subjects read upside-down passages faster 1 year after their initial training session. Future studies should test whether the same statistical learning mechanism underlies contextual cuing and Kolers's "patternanalyzing memory." We should also directly test the utility of contextual cuing in real-world spatial navigation and visual perception.

\section{REFERENCES}

Anderson, M. C., \& Neely, J. H. (1996). Interference and inhibition in memory retrieval. In E. L. Bjork \& R. A. Bjork (Eds.), Memory (pp. 237-313). San Diego: Academic Press.

Chapman, L. J., Chapman, J. P., Curran, T. E., \& Miller, M. B. (1994). Do children and elderly show heightened semantic priming? How to answer the question. Developmental Review, 14, 159-185.

Chun, M. M., \& JiAnG, Y. (1998). Contextual cueing: Implicit learning and memory of visual context guides spatial attention. Cognitive Psychology, 36, 28-71.

Chun, M. M., \& Jiang, Y. (1999). Top-down attentional guidance based on implicit learning of visual covariation. Psychological Science, 10, 360-365.

Chun, M. M., \& Jiang, Y. (2003). Implicit, long-term spatial contextual memory. Journal of Experimental Psychology: Learning, Memory, \& Cognition, 29, 224-234.

Crowder, R. G. (1976). Principles of learning and memory. Hillsdale, NJ: Erlbaum.

ENDO, N., \& TAKEDA, Y. (2004). Selective learning of spatial configuration and object identity in visual search. Perception \& Psychophysics, 66, 293-302.

FISER, J., \& AsLIN, R. N. (2001). Unsupervised statistical learning of higher-order spatial structures from visual scenes. Psychological Science, 12, 499-504.

JACOBY, L. L. (1983). Perceptual enhancement: Persistent effects of an experience. Journal of Experimental Psychology: Learning, Memory, \& Cognition, 9, 21-38.

Kolers, P. A. (1976). Pattern analyzing memory. Science, 191, 12801281.

Lassaline, M. E., \& Logan, G. D. (1993). Memory-based automaticity in the discrimination of visual numerosity. Journal of Experimental Psychology: Learning, Memory, \& Cognition, 19, 561-581.

Levin, D. T., \& Simons, D. J. (1997). Failure to detect changes to attended objects in motion pictures. Psychonomic Bulletin \& Review, 4, 501-506.

LogAN, G. D. (1988). Toward an instance theory of automatization. Psychological Review, 95, 492-527.

Lustig, C., \& HaSHLER, L. (2001a). Implicit memory is not immune to interference. Psychological Bulletin, 127, 629-650.
Lustig, C., \& Hashler, L. (2001b). Implicit memory is vulnerable to proactive interference. Psychological Science, 12, 408-412.

OLSON, I. R., \& CHUN, M. M. (2001). Temporal contextual cueing of visual attention. Journal of Experimental Psychology: Learning, Memory, \& Cognition, 27, 1299-1313.

OsGood, C. E. (1953). Method and theory in experimental psychology. New York: Oxford University Press.

Rensink, R. A. (2002). Change detection. Annual Review of Psychology, 53, 245-277.

Rensink, R. A., O'Regan, J. K., \& Clark, J. J. (1997). To see or not to see: The need for attention to perceive changes in scenes. Psychological Science, 8, 368-373.

SCHACTER, D. L. (1996). Searching for memory: The brain, the mind, and the past. New York: Basic Books.

Schneider, W., \& Shiffrin, R. M. (1977). Controlled and automatic human information processing: I. Detection, search, and attention. Psychological Review, 84, 1-66.

Simons, D. J., \& Levin, D. T. (1998). Failure to detect changes to people during a real-world interaction. Psychonomic Bulletin \& Review, 5, 644-649.

Tulving, E., \& Schacter, D. L. (1990). Priming and human memory systems. Science, 247, 301-306.

\section{NOTES}

1. We also analyzed median RT and outlier-trimmed mean RT $(\mathrm{RT}<$ $5 \mathrm{sec}$ ). These measures gave patterns of results similar to mean RT. Mean RT without trimming was reported here because performance became stable with practice.

2. Whether one uses data from the last 20 blocks, the last 15 blocks, or the last 5 blocks does not affect the pattern of results. We chose to report data from blocks 11-30 because previous studies show that learning emerges relatively quickly; by the 11 th block, a clear learning effect has already emerged.

3. When all data were entered in an ANOVA, with day (1-5), condition (new or old), and block (1-30) as factors, we observed significant main effects of all factors and a significant interaction between day and block. Thus, RT became faster with practice, and this improvement was bigger on earlier days.

4. We also fitted a power function to relate RT with block. In all sessions, the old condition was fitted with a steeper slope than was the new one.

5. Note that this correlation analysis is highly sensitive: Even when each pairwise correlation was low (e.g., $r=.05$ ), as long as the 10 pairwise correlations were mostly positive, the one-sample $t$ test of these 10 values would be significant. The lack of significance means that the pairwise correlation was highly inconsistent, sometimes positive and sometimes negative.

6. We also analyzed the data of Session 6 with the data of the first and second blocks separated. Overall, the second block was responded to faster than was the first $(p<.05)$, but the size of contextual cuing was comparable in the two blocks $(p>.15)$.

(Manuscript received May 17, 2004; revision accepted for publication September 5, 2004.) 